\title{
Antropometría del trabajador minero de la altura
}

\author{
Augusto V Ramírez ${ }^{1}$
}

"El hombre es la medida de todas las cosas, de las que son en cuanto son y de las que no son en cuanto no son".

Protágoras de Abdera 458-411 a.C.

Resumen

Palabras clave
Introducción: La antropometría trata de las medidas del cuerpo humano y es base fundamental para la ergonomía. La minería peruana se localiza casi siempre en la gran altura, hábitat del hombre andino, que tiene características antropométricas diversas al hombre del llano. Objetivo: Caracterizar antropométricamente a una población andina de trabajadores mineros en la sierra central del Perú. Diseño: El estudio fue descriptivo, tipo prospectivo y observacional. Lugar: Servicio de Salud Ocupacional, Centromin-Perú, en La Oroya, y 4 de sus hospitales mineros satélites. Participantes: Tres mil trabajadores mineros, sanos, de sexo masculino, de 21 a 60 años, nativos-residentes en alturas superiores a 3500 msnm. Intervenciones: La muestra la generamos con un modelo aleatorio polietápico de conglomerados. El estudio se realizó entre 1984-1994; obtuvimos media, rango y desviación estándar de la media. Para la certeza estadística de la hipótesis de normalidad, aplicamos la prueba de significancia de Shapiro-Wilk, para dos colas. Principales medidas de los resultados: Medidas morfológicas, macroscópicas, fenotípicas y de superficie, realizadas con un protocolo de medicióny según técnica reconocida en el ámbito científico. Resultados: De los parámetros estudiados destacaron: de pie, estatura 151,2 a 154,6, altura piso-ojos, 1,481 m; sentado, asiento-codo 0,243 m, a vértex y altura ojos, 1,155 y 1,095 m; mano, ancho y largo, 0,104 a 0,171 m; pie, ancho y largo, 0,92 y 0,239 m; perímetro tórax, reposo 98,16, inspiración forzada, 105,3, espiración forzada 94,3 $\mathrm{cm}$. Los resultados fueron validados con la prueba de Shapiro Wilk que, para aceptación, valorax entre 0,01 y 0,099. Del total (47), tres parámetros fueron rechazados totalmente: alcance vertical máximo de brazo, ancho de caderas y perímetro de cráneo, cuyo valor de x en los tres casos fue $\geq 0,50$. Conclusiones: La caracterización antropométrica del trabajador minero andino resalta sus dimensiones propias, que le hacen ser un modelo humano diferente al del llano.

Antropometría; antropología física; altitud; ergonomía; minería.

\begin{abstract}
High altitude mining workers anthropometry Abstract

Introduction: Anthropometry is the essential basis for ergonomics, mainly human body's measures referred to size and shapes. Peruvian mining is located at high altitudes, habitat of ethnic Andean men showing different anthropometry to the sea level man. Objectives: To characterize anthropometrics of a Peruvian Andean Mountain mining workers, native men population, residing and living at 3000 masl and more. Design: Descriptive, prospective and observational study. Setting: La Oroya Centromin-Peru Enterprise's Occupational Health Service and its four mining satellite hospitals. Participants: Three thousand healthy native 21 to 60 year-old male mining workers residing in altitudes above 300 masl. Interventions: The sample was generated by conglomerate's random aleatory polyphase model. The study was done between 1984-1994. For normality hypothesis
\end{abstract}

\footnotetext{
1 American College of Occupational and Environmental Medicine.
}

statistical certainty we applied Shapiro-Wilk's two tails significant test. Main outcome measures: Morphologic, macroscopic, phenotypic and surface measurements done with a measurement protocol and according to a scientific recognized technique. Results: Highlighted parameters were: standing, stature 151,2 to 154,6, floor-eyes height, 1,481 m; sitting, chair-elbow 0,243 m, to vertex and eye height, 1,155 and 1,095 m; hand, width and length, 0,104 to 0,171 m; foot, width and length, 0,92 and 0,239 m; thoracic perimeter, resting 98,16, forced inspiration, 105,3, forced espiration, 94,3 cm. Results were validated with Shapiro Wilk's test that values $x$ between 0,01 and 0,099. From the whole (47), three parameters were totally rejected: arm maximum vertical reach, hip width and cranium perimeter, whose parameters $x$ value was $\geq 0,50$. Conclusions: The Andean miner worker has proper dimensions different from the sea level man. Integral anthropometric and ergonomics parameters investigations on high altitude living Andean workers should be carried out.

Key words: Anthropometry; anthropology, physical; altitude; ergonomics; mining. 


\section{INTRODUCCIÓN}

La revolución industrial iniciada hace dos siglos trajo aparejados cambios tecnológicos y de los sistemas de trabajo, que han llevado al mundo al avance actual en que el hombre alcanza un desarrollo inusitado. Cuenta hoy con herramientas, equipos, máquinas y toda laya de tecnología cuya utilidad tiene como confesa finalidad la búsqueda de confort y bienestar en su vida diaria, y eficacia, adaptabilidad, prevención y seguridad en el trabajo. Naturalmente, estos adelantos llegan siempre de la mano con nuevos modelos de equipos, máquinas, herramientas o vehículos, a cual más disímiles, que fuerzan al hombre a acomodarse dentro o fuera de ellos, lo que implica incremento de riesgos, sobre todo en el trabajo $\left(^{1}\right)$.

La antropometría es la ciencia que trata de las medidas del cuerpo humano, principalmente las que se refieren a su tamaño, al tamaño de sus segmentos, formas, fuerza y capacidad de trabajo y es una de las bases fundamentales de la ergonomía. La ergonomía es una técnica de aplicación práctica, interdisciplinaria y fundamentada en investigación científica cuyo objetivo es la optimización integral de los sistemas 'hombre-máquina', compuestos por uno o más seres humanos que realizan una tarea cualquiera con ayuda de una o más máquinas. 'Máquina', aquí, es un término genérico aplicado a todo tipo de equipos, herramientas, máquinas industriales propiamente dichas, vehículos, computadoras, electrodomésticos, etc. $\left({ }^{2}\right)$.

En este contexto, la ergonomía usa los datos antropométricos para diseñar espacios de trabajo, herramientas, equipos de seguridad y protección personal, considerando las diferencias entre las características, capacidades y límites físicos del cuerpo humano. Existen, hoy en día, diversos estudios ergonómicos orientados al control de los riesgos laborales originados por la no adaptación de los equipos de trabajo a las medidas del ser humano $\left({ }^{3}\right)$. Se sigue que, la antropometría es la determinante de las condiciones ergonómicas; por tanto, los estudios antropométricos deben referirse a una población específica y de ahí nuestro interés por conocer las medidas de la población trabajadora andina $\left({ }^{4,5}\right)$

Los antepasados de nuestro actual hombre andino han vivido a más de $4000 \mathrm{msnm}$ no menos de 10000 años, hábitat que supone ante todo condiciones hipóxicas de sobre vivencia. Esta larga permanencia en las cumbres andinas les ha llevado a una aclimatación exitosa; y, tareas que demanden esfuerzo físico vigoroso o actividad intelectual, las realizan con igual eficacia que un trabajador del llano $\left(^{6}\right)$.

La población peruana actual es mestiza y étnicamente está constituida por diferentes grupos: nativos (45\%), mestizos-nativos con ancestros de europeos (37\%), blan$\cos (15 \%)$, negros, japoneses, chinos y otros $(3 \%)\left({ }^{7}\right)$. A pesar de la predominancia de nativos y mestizos nativos, son muy escasos los estudios antropométricos del trabajador peruano y menos de nativos andinos, que constituyen la población laboral mayoritaria en la minería del Perú.

Nuestras poblaciones nativas andinas han sido estudiadas desde el siglo XIX. Al principio fueron antropólogos y biólogos y luego fisiólogos. El interés aún hoy es grande, porque el continente americano desde su descubrimiento representa, en el real sentido de la palabra, un 'mundo nuevo' poblado muy recientemente por hombres provenientes de Asia que cruzaron el estrecho de Behring en grupos pequeños $\left(^{8}\right)$.

Vellard, citado por Spielvogel, sostiene que América se pobló en sucesivas migraciones y mediante olas de dos grupos de poblaciones. Unos se asentaron circunvecinos al lago Titicaca, por lo que les llama paleoamerindios 'láguidos' y cuyos descen- 
dientes actuales son los uros. Al otro grupo, neoamerindios, Vellard los divide en altiplánidos y ándidos. Los altiplánidos, kollas, forman el Tiahuanacu, hablan el aymará y se asientan en la hoy Bolivia. Los ándidos, más al noroeste, forjan el imperio Inca e imponen el quechua como lengua. Al final, y como resultado de estos inicios migratorios, ambas poblaciones andinas permanecen aisladas en la altiplanicie de sur América hasta bien entrado el milenio anterior, cuando los incas inician su expansión, llegando primero a tribus circunvecinas y luego a toda la región andina y costera de sudamérica e inclusive a parte de nuestra selva amazónica $\left({ }^{9}\right)$.

Muchos de los estudios en nativos del ande peruano realizados desde fines de $s$. XIX demostraron ya que su perímetro torácico y la capacidad de sus pulmones eran mayores que los del hombre del llano. Otro punto interesante y significativo es su menor talla, respecto del habitante de la costa. La explicación aceptada es que la menor talla está relacionada con una maduración retrasada, mientras que el aumento del perímetro del tórax y la capacidad pulmonar resulta de una adaptación funcional a la difícil respiración en nuestra gran altura, mecanismo adaptativo que por cierto sería diferente del nativo de otras alturas, como las del Himalaya $\left({ }^{10}\right)$.

Por estas cuestiones de adaptación, el hombre andino tiene un modelo somático diferente al $H$. sapiens del llano, pues la necesidad de residir en la altiplanicie andina le llevan a desarrollar características propias que le permitan vivir en esas condiciones ambientales particulares. El ambiente andino, y el tiempo, le han dejado entre otras huellas la amplitud del tórax, la forma del tronco y su talla. Respecto a definir quiénes son los adaptados a vivir en los Andes, grandes fisiólogos peruanos del hombre de altura, con Monge Medrano en primer lugar, han sentenciado "Nuestra experiencia nos ha llevado a plantear que la óp- tima calidad de aclimatación a 'las alturas habitables' se encuentra en nativos indígenas, apegados a su estilo de vida ancestral" $\left({ }^{11-13}\right)$.

Siendo diferente como es el hombre andino, fundamental es conocer literalmente cuánto mide. Pero, entonces nosotros, peruanos y mineros conocemos cuánto mide en el amplio sentido de la palabra el hombre de nuestros Andes? Indudablemente, cualquier respuesta queda corta; y por eso nuestra investigación en un grupo representativo de trabajadores minero-metalúrgicos de los andes centrales del Perú pretende desvelar, en medida inicial, estas interrogantes, lo que nos servirá de guía para aplicar un sistema ergonómico básico.

Nuestro estudio, de carácter observacional y prospectivo, lo realizamos con protocolo propio en el Departamento de Medicina Ocupacional y en los hospitales mineros de Cerro de Pasco, Casapalca, Morococha y Yauricocha, de la Empresa Minera del Centro del Perú S.A., en el lapso 1984 - 1 994. Como resultado de esta investigación, conocemos ahora las magnitudes de las líneas antropométricas fundamentales y los parámetros ergonómicos de mayor significado para el trabajador minero nativo de los andes centrales del Perú. Confirmamos, además de la conocida amplitud de su tórax, que en el grupo de 21 a 60 años la talla promedio es de $158,4 \mathrm{~cm}$. y que su peso está en el rango de 50,4 a $71,5 \mathrm{~K}$. Estos hallazgos nos permitirán, entre otras cosas, adecuar la máquina a nuestros trabajadores, mejorando así sus condiciones de labor. Sin embargo, el campo de investigación ergonómica en el trabajador de altura aún es vasto.

\section{MATERIALES Y MÉTODOS}

Nuestro objetivo fundamental fue caracterizar antropométricamente a una población andina de trabajadores mineros de la 
sierra central del Perú, para obtener una base de referencia. El estudio de 47 parámetros antropométricos lo desarrollamos mediante investigación descriptiva de tipo prospectivo observacional, generando la muestra por polietapas de conglomerados $\left({ }^{14}\right)$.

El estudio abarca al $46 \%$ de los 6500 trabajadores mineros y metalúrgicos andinos pertenecientes a la planilla de la Empresa Minera del Centro del Perú S.A. (Centromín Perú), sanos, de sexo masculino y edades entre 21 y 60 años, hijos de nativos y ellos mismos nativos y residentes en alturas iguales o superiores a $3000 \mathrm{msnm}$, procedentes de los departamentos de Junín, de Cerro de Pasco y de la sierra de Lima. La zona de origen y hábitat de la población estudiada se ubica geográficamente entre $\operatorname{los} 9^{\circ} 31^{\prime}$ y $\operatorname{los} 13^{\circ} 30^{\prime}$ de latitud sur y desde el punto de vista altimétrico y geomorfológico, la población proviene de las regiones Suni, Jalca y Puna, del Altiplano Andino, localizadas entre 3500 y 4800 msnm $\left({ }^{15,16}\right)$.

Nuestra investigación focaliza a los trabajadores de la Empresa Minera del Centro del Perú, entre los cuales realizamos la selección por método aleatorio individual e incluimos a los trabajadores que cumplían estrictamente con lo descrito en el marco muestral.

Excluimos a todo trabajador que en cualquier etapa de su vida había salido a residir permanentemente por más de 2 años a nivel de altitud menores a $3000 \mathrm{msnm}$ y a los que al momento del examen no estuviesen clínicamente sanos, tuviesen amputaciones de miembros o segmentos de miembros y limitaciones articulares o posturales.

Otro criterio de exclusión se dio en la conformación de los grupos etáreos, pues para evitar posibles sesgos por desarrollo físico incompleto, iniciamos la muestra del primer grupo en los 21 años cumplidos.
Decidimos también que cada grupo debería ser proporcional a la población total actual de trabajadores, por lo que la mayor parte la distribuimos en los grupos de 31 a 50 años, que en la Empresa constituían cerca del $65 \%$ de la fuerza laboral.

La población estudiada cumple con la definición de nativos $\left({ }^{11}\right)$. El estilo de vida $\mathrm{y}$ actividades del universo estudiado corresponden a las del minero o metalurgista andino peruano que muy ocasionalmente, y cada vez menos, en la época de lluvia deriva por breve lapso a agricultor tradicional en su mismo hábitat o muy cerca a él, pero nunca a menos de $3000 \mathrm{msnm}$.

Todos los trabajadores estudiados se desarrollaron físicamente en la altura, son residentes permanentes en ella y todos tenían por lo menos un año de experiencia laboral y dependían directamente de la empresa.

El estudio se desarrolló en el lapso 1984 - 1994 en el Servicio de Salud Ocupacional de la empresa en la Oroya y en 4 de sus hospitales mineros. La evaluación la iniciamos en 1984 y fue realizada concomitante al examen médico ocupacional que a cada uno de los trabajadores se le practica periódicamente de acuerdo a la ley de minería. El año de 1994, la concluimos.

La evaluación, de acuerdo a estándares internacionales, considera como dimensiones antropométricas a las medidas morfológicas, macroscópicas, fenotípicas y de superficie, realizadas a las personas con un protocolo de medición y según técnica reconocida en el ámbito científico $\left({ }^{17}\right)$. Además de datos antropológicos usuales, medimos 11 líneas antropométricas fundamentales y 36 parámetros ergonómicos básicos. La colaboración de cada trabajador en el desarrollo del estudio fue esencial, pues, como además se trataba de su examen clínico ocupacional anual, le pedimos se desvistiera completamente y se quitara los zapatos. 
Iniciamos el examen con una instrucción detallada de lo que esperábamos de cada uno, luego procedimos con la medición. Cada medida fue tomada dos veces y si entre ellas había diferencia mayor a la tolerancia pre establecida $(3 \mathrm{~mm})$, procedimos a una tercera medición y registramos la media. Toda magnitud que implicaba medida de un lado del cuerpo fue realizada en el lado derecho.

En la toma de las medidas se adoptó las siguientes posiciones:

1. Parado erguido: el trabajador permaneció parado y erguido, mirando hacia el frente, los tobillos juntos, el peso distribuido equitativamente en ambos pies y los brazos colgando naturalmente sueltos a los lados.

2. Sentado erguido: aquí el trabajador estaba sentado, pero con el tronco erguido, con la vista hacia el frente, los brazos colgando relajados, antebrazos y manos extendidas hacia adelante, los muslos horizontales y pies descansando sobre el piso, de manera tal que las rodillas quedaran flexionadas en ángulo de $90^{\circ}$

Los resultados fueron expresados en magnitudes del sistema métrico:

Peso en kilos y altura y otras magnitudes -como anchuras, larguras y perímetros- en centímetros.

En el registro de peso usamos balanzas mecánicas de pie del tipo 'médica' (una por sede), con resolución de $100 \mathrm{~g}$, que fue calibrada inicialmente usando su propio sistema de calibración y luego recalibrada cada día que realizábamos las mediciones.

Para registrar talla de pie, usamos un estadiómetro, fabricado con 3 planos de madera, 2 horizontales y un larguero vertical, cruzados en ángulo recto. Al plano vertical adherimos una cinta metálica rígida (wincha) de $1 \mathrm{~cm}$ de ancho y $2 \mathrm{~m}$ de largo.
El plano horizontal superior móvil se desplazaba arriba abajo en un eje acanalado del plano vertical, de manera tal que, al colocarlo sobre la cabeza del trabajador, pudiésemos leer su estatura en la intersección.

Usamos la técnica de "la altura en extensión máxima (stretch stature)", que requiere medir la máxima distancia entre el piso y el vértex. Para ello, la posición de la cabeza debe estar en el plano de Frankfort, es decir, el arco orbital inferior debe ser alineado horizontalmente con el trago, formando una línea imaginaria perpendicular al eje longitudinal del cuerpo. Se indicaba al trabajador que sin levantar la cabeza mirara a un punto imaginario directamente frente a él. El evaluador se ubicaba delante y le pidió colocar pies y rodillas juntas; talones, cara posterior de glúteos y cabeza pegados al plano posterior del estadiómetro. Luego, por debajo de la mandíbula, con ambas manos tomaba la cabeza por los costados y le pedía que respirara hondo y levantándosela suavemente le indicaba se relajara y estirara. En ese momento, colocaba el plano móvil superior sobre el vértex y leía el valor de la talla en el plano vertical.

En el registro de talla en posición sentado medimos la distancia entre el vértex y el plano de un asiento, apoyado contra el plano vertical del estadiómetro (banco de madera de altura regulable, asiento redondo de $25 \mathrm{~cm}$ de diámetro). Para la medición, usamos una cinta métrica accesoria, cuyo cero coincidía con el plano superior del asiento del banco; sentamos al trabajador con pies y rodillas juntos y realizamos el mismo procedimiento que para el registro de la talla de pie. En ambos casos, revisamos la nivelación de los planos y la cinta métrica cada 50 mediciones.

Registramos los perímetros usando una cinta métrica de las llamadas 'de sastre', de $0,5 \mathrm{~cm}$ de ancho y $1,5 \mathrm{~m}$ de largo, flexible pero no extensible, con resolución de lectura de $0,1 \mathrm{~cm}$, la cual era de fácil mani- 
pulación. Durante todas las mediciones, la cinta fue sostenida a la usanza del sastre, por el cuarto y quinto dedo de la mano derecha; esto nos permitía: 1) que los pulgares e índices de ambas manos controlasen el grado de tensión y alineamiento de la cinta sobre la piel del trabajador; y, 2) verificar que la cinta no quedase floja, doblada, fuera de contacto de la piel y que no comprimiese el contorno a medir. Luego la otra mano colocaba el extremo inicial sobre el extremo final de la cinta y objetivamos la magnitud en la intersección de ambos segmentos $\left({ }^{17-18}\right)$.
Los diámetros internos los medimos con pelvímetro de resolución al milímetro $\mathrm{y}$, a fin de estandarizar las mediciones, definimos los criterios para describir los parámetros a estudiar y los incluimos en la Tabla 1.

En la evaluación estadística de las variables estudiadas, obtuvimos el promedio (media), el rango (valores extremos) y la desviación estándar de la media (DEM) de cada medición. Para tener certeza estadística, a la totalidad de las medidas obtenidas las evaluamos para la hipótesis de nor-

Tabla 1. Definiciones.

\section{Líneas antropométricas fundamentales}

Yugulo xifoidea. Distancia recta entre la horquilla del esternón y la punta del apéndice xifoides.

Epigástrico pubiana. Distancia recta del epigastrio al pubis.

Xifo-epigástrica. Distancia de apéndice xifoides y el epigastrio.

Miembro superior (hasta la muñeca). Distancia desde el nacimiento del brazo (hombro) a la apófisis estiloides de la muñeca con el brazo extendido.

Miembro inferior (hasta el maléolo). Distancia del trocánter al maléolo.

Diámetro transverso del tórax. Distancia longitudinal anteroposterior del tórax medida a la altura de las mamilas.

Diámetro anteroposterior del tórax. Distancia interna del tórax medida entre el esternón y la $6^{\text {a }}$ dorsal.

Diámetro transverso hipocondríaco. Distancia longitudinal anteroposterior del tórax medida a la altura de los hipocondrios.

Diámetro anteroposterior hipocondríaco. Distancia interna del tórax medida a la altura de los hipocondrios.

Diámetro bi-crestílico. Distancia interna medida entre las crestas iliacas $\left({ }^{18}\right)$.

\section{Parámetros ergonómicos}

Estatura (talla). Es la distancia vertical desde el suelo al vértex, tomada en una persona de pié, erguida y con la vista dirigida al frente. Altura de piso a ojos. Distancia vertical del piso hasta la comisura exterior del ojo, tomada a una persona de pié, erguida y con la vista al frente.

Altura de piso a hombros. Distancia vertical de piso a hombro en posición de pié.

Altura de piso a codos. Altura que va del piso a la unión de brazo y antebrazo.

Alcance mínimo del brazo. Distancia horizontal desde el respaldo del asiento hasta el eje vertical de la mano con el puño cerrado y el brazo paralelo a la línea media del tronco y el antebrazo forma un ángulo de $90^{\circ} \mathrm{con}$ el brazo.

Alcance máximo del brazo hacia delante. Distancia horizontal desde el respaldo del asiento hasta el eje vertical, que se produce en la mano con el puño cerrado y sosteniendo un eje, cuando el individuo tiene el brazo extendido.

Ancho de codo a codo. Distancia que separa las superficies laterales de los codos, medida cuando están doblados, ligeramente apoyados al cuerpo.

Altura de codo desde el asiento. Distancia vertical desde el asiento hasta el codo en posición de reposo a $90^{\circ}$.

Altura de muslo desde el asiento. Distancia vertical desde la superficie del asiento hasta la parte superior del muslo, junto al abdomen. Distancia sacro-poplítea. Distancia horizontal desde la parte exterior del sacro hasta la cara posterior de la rodilla.

Distancia sacro-rótula. Distancia horizontal desde la parte exterior del sacro hasta la cara anterior de la rótula.

Ancho de caderas. Es la mayor medida horizontal entre las caderas y sentado.

Altura poplítea. Distancia vertical desde el suelo hasta la zona inmediatamente posterior de la rodilla con el individuo sentado, tronco erguido y piernas a $90^{\circ}$

Altura del muslo desde el suelo. Distancia vertical con el sujeto sentado desde el suelo hasta la parte superior del muslo.

Miembro inferior (hasta el maléolo). Distancia vertical que va de raíz del muslo a maléolo externo $\left({ }^{18}\right)$. 
malidad, con la prueba de significancia de Shapiro - Wilk para dos colas $\left({ }^{19}\right)$.

\section{RESULTADOS}

Los 3000 trabajadores estudiados, pertenecientes a Centromin Perú S.A., fueron nativos de la altura, todos de sexo masculino y laboraban en planilla con las siguientes características demográficas. Por lugar de nacimiento, residencia y labor, los trabajadores provenían de los departamentos de Lima (minas y concentradoras de Yauricocha a $4654 \mathrm{msnm}$ y Casapalca a $4400 \mathrm{msnm}$ ), Pasco (Cerro de Pasco mina y concentradora, a $4340 \mathrm{msnm}$ ) y Junín (Fundición La Oroya, $3735 \mathrm{msnm}$, y Morococha, mina y concentradora a 4500 msnm).

La descripción del universo estudiado por procedencia y por tipo de labor la presentamos en la Tabla 2.

Tabla 2. Número de estudiados, por ciudad de procedencia del universo, por tipo de labor. Sexo: masculino.

\begin{tabular}{lrrrrr}
\hline \multicolumn{1}{c}{ Mina } & $\begin{array}{c}\text { Altura } \\
\text { msnm }\end{array}$ & $\mathrm{n}$ & $\%$ & \multicolumn{2}{c}{$\begin{array}{c}\text { Ocupación } \\
\text { Minero }\end{array}$} \\
& 3750 & 1000 & 33,3 & 0 & 1000 \\
\hline La Oroya (Junín) & 3400 & 100 \\
Cerro de Pasco (Pasco) & 4340 & 800 & 26,9 & 700 & 100 \\
Morococha (Junín) & 4450 & 400 & 13,3 & 300 & 100 \\
Yauricocha (Lima) & 4654 & 400 & 13,3 & 400 & 0 \\
Casapalca (Lima) & 4267 & 400 & 13,3 & 200 & 200 \\
Total & & 3000 & 100 & 1600 & 1400 \\
\hline
\end{tabular}

Metal. $=$ Metalúrgico.

msnm = Ubicación geográfica de la mina en metros sobre el nivel del mar.

La distribución por grupos de edad la observamos en la Tabla 3.

En la Tabla 4 mostramos los resultados de las características antropológicas generales de la población estudiada, como edad, talla, peso perímetro torácico y craneal, tratadas estadísticamente con media, rango y desviación estándar.
Tabla 3. Distribución por grupo etáreo.

\begin{tabular}{lrr}
\hline Edad en años & $\mathrm{n}$ & \multicolumn{1}{c}{$\%$} \\
\hline $21-30$ & 500 & 16,6 \\
$31-40$ & 1100 & 36,7 \\
$41-50$ & 1100 & 36,7 \\
$51-60$ & 300 & 10 \\
Total & 3000 & 100 \\
\hline
\end{tabular}

Las Tablas 5 y 6 presentan las medias, rangos, desviaciones estándar, análisis estadístico y valor de aceptación o rechazo del parámetro, con la prueba de ShapiroWilk. De los parámetros estudiados, destacan por su significancia en ergonomía -además de estatura-, altura piso a ojos, 1,481 $\mathrm{m}$; altura de asiento a codo $0,243 \mathrm{~m}$; sentado, altura a vértex y a ojos, 1,155 y 1,095 $\mathrm{m}$; ancho y largo de mano, 0,104 y 0,171 $\mathrm{m}$; ancho y largo de pie, 0,92 y 0,239 m; perímetro de tórax, en reposo, 98,16, en inspiración forzada, 105,3, y en espiración forzada, 94,3 cm, respectivamente. Estos y otros parámetros fueron validados (aceptados) por la prueba de Shapiro Wilk, con un valor de $x$ entre 0,01 y 0,099. Sin embargo, también es necesario indicar que tres (3) parámetros -alcance vertical máximo de brazo, ancho de caderas y perímetro de cráneo- fueron rechazados por la prueba con valor de $x \geqslant$ de 0,50 , por lo que sensu estricto sus valores no deberían ser tomados en cuenta.

Tabla 4. Antropometría del trabajador minero de la altura. Características antropométricas generales de la población estudiada $(\mathrm{n}=3)$.

\begin{tabular}{llccr}
\hline \multicolumn{1}{c}{ Parámetro } & \multicolumn{1}{c}{ Unidad } & Media & Rango & DEM \\
\hline Edad & Años & 36,7 & $21-60$ & \\
Talla & Centímetros & 158,4 & $151,2-169,5$ & 2,37 \\
Peso & Kilos & 58,7 & $50,4-71,5$ & 7,3 \\
Perímetro tórax: & & & & \\
$\quad$ Reposo & Centímetros & 98,16 & $94,2-104,5$ & 3,7 \\
$\quad$ Inspiración forzada & Centímetros & 105,3 & $99,7-108,6$ & 1,8 \\
$\quad$ Espiración máxima & Centímetros & 94,3 & $90,9-99$ & 2,1 \\
Perímetro cráneo & Centímetros & 56,08 & $55,3-57,01$ & 1,9 \\
\hline
\end{tabular}


Tabla 5. Antropometría del trabajador minero de la altura. Análisis estadístico de las líneas fundamentales.

\begin{tabular}{|c|c|c|c|c|}
\hline $\begin{array}{l}\text { Línea antropométrica } \\
\text { (centímetros) }\end{array}$ & Media & VE & DEM & $\begin{array}{l}\text { P Shapiro } \\
\text { Wilk* }\end{array}$ \\
\hline Yúgulo xifoidea & 20,6 & $20,10-20,93$ & 1,1 & A \\
\hline Epigástrico pubiana & 20,5 & $19,00-21,20$ & 1,8 & A \\
\hline Xifo epigástrica & 10,5 & 9,75-10,99 & 2,2 & A \\
\hline $\begin{array}{l}\text { Miembro superior } \\
\text { (hasta la muñeca) }\end{array}$ & 53,0 & $52,25-53,56$ & 1,7 & A \\
\hline $\begin{array}{l}\text { Miembro inferior } \\
\text { (hasta el maléolo) }\end{array}$ & 73,3 & $71,45-78,00$ & 3,8 & $\mathrm{R}$ \\
\hline $\begin{array}{l}\text { Diámetro transverso } \\
\text { del tórax }\end{array}$ & 26,6 & $25,20-27,10$ & 0,5 & A \\
\hline $\begin{array}{l}\text { Diámetro bitrocantéreo } \\
\text { Diámetro antero }\end{array}$ & 29,0 & $26,70-30,90$ & 1,8 & A \\
\hline $\begin{array}{l}\text { posterior del tórax } \\
\text { Diámetro transverso }\end{array}$ & 20,6 & $19,85-21,84$ & 1,4 & A \\
\hline $\begin{array}{l}\text { hipocondríaco } \\
\text { Diámetro antero }\end{array}$ & 24,5 & $23,60-26,40$ & 2,4 & $\mathrm{R}$ \\
\hline posterior hipocondríaco & 18,6 & $17,82-19,51$ & 2,4 & A \\
\hline Diámetro bi-crestílico & 29,1 & $28,20-29,70$ & 2,4 & A \\
\hline
\end{tabular}

VE: Valores extremos

DEM: Desviación estándar de la media

Prueba Shapiro Wilk:

Nivel de rechazo valor $p<$ dos colas

Representación en la Tabla:

$\begin{array}{ll}A=\text { Aceptación }: & \text { Valor } x: 0,01 \text { a } 0,099 \\ R=\text { Rechazo: } & \text { Valor } x: 0,10 \text { a } 0,49 \\ R F=\text { Rechazo fuerte: } & \text { Valor } x:>0,50\end{array}$

Finalmente, la Tabla 7 nos muestra pormenorizados los resultados de la evaluación del parámetro talla, cuya media es 1,584 , con valores extremos de 1,512 y $1,695 \mathrm{~m}$, desviación estándar de 2,37 y error estándar de 0,17.

Para una mejor visualización, estos mismos parámetros los presentamos diagramados en la Figura 1.

\section{DISCUSIÓN}

Desde la época colonial, el Perú es un país minero por antonomasia y la minería es su actividad industrial básica. Las operaciones mineras se encuentran ubicadas entre los 3000 y $5000 \mathrm{msnm}$, zonas denominadas por cuestiones fisiológicas como
Tabla 6. Antropometría del trabajador minero de la altura. Análisis estadístico de los parámetros ergonómicos.

\begin{tabular}{|c|c|c|c|c|}
\hline $\begin{array}{c}\text { Parámetro } \\
\text { (centímetros) }\end{array}$ & Media & $\mathrm{VE}$ & DEM & $\begin{array}{l}\text { P Shapiro } \\
\text { Wilk* }\end{array}$ \\
\hline Estatura & 158,4 & $151,2-169,5$ & 2,37 & A \\
\hline Altura de piso a ojos & 148,1 & $141,4-155,1$ & 0,52 & A \\
\hline $\begin{array}{l}\text { Altura de piso } \\
\text { acromion }\end{array}$ & 135,0 & $129,0-143,1$ & 2,35 & A \\
\hline Altura de piso a codo & 65,3 & $62,3-65,7$ & 3,01 & A \\
\hline Altura sentado & 115,5 & $110,6-125,3$ & 2,18 & A \\
\hline Altura ojos, sentado & 109,5 & 107,7-111,1 & 1,45 & A \\
\hline $\begin{array}{l}\text { Altura de asiento a codo } \\
\text { Altura fosa poplítea }\end{array}$ & 24,3 & $23,4-25,0$ & 0,90 & A \\
\hline $\begin{array}{l}\text { sentado } \\
\text { Altura a muslo desde }\end{array}$ & 41,1 & $39,2-43,9$ & 3,06 & A \\
\hline $\begin{array}{l}\text { asiento (sentado) } \\
\text { Altura a muslo desde }\end{array}$ & 13,0 & $12,5-15,0$ & 0,40 & A \\
\hline $\begin{array}{l}\text { suelo (sentado) } \\
\text { Alcance vertical }\end{array}$ & 54,0 & $52,8-55,3$ & 2,65 & A \\
\hline $\begin{array}{l}\text { máximo de brazo } \\
\text { Alcance vertical }\end{array}$ & 207,2 & $202,1-220$ & 4,93 & $\mathrm{RF}$ \\
\hline $\begin{array}{l}\text { mínimo de brazo } \\
\text { Alcance máximo }\end{array}$ & 201,9 & $197,0-113,3$ & 1,01 & A \\
\hline $\begin{array}{l}\text { brazo hacia delante } \\
\text { Alcance mínimo }\end{array}$ & 73,8 & $69,9-78,4$ & 2,05 & A \\
\hline brazo hacia delante & 43,0 & $39,5-47,0$ & 1,16 & A \\
\hline Ancho mano & 10,4 & $9,0-12,1$ & 0,96 & A \\
\hline Largo de mano & 17,1 & $16,5-18,1$ & 1,70 & A \\
\hline Ancho pie & 9,2 & $8,9-9,4$ & 2,30 & A \\
\hline Largo pie & 23,9 & $22,7-24,4$ & 2,91 & A \\
\hline Ancho de caderas & 36,5 & $34,5-38,0$ & 4,74 & $\mathrm{RF}$ \\
\hline Distancia sacro-poplítea & 46,0 & $43,0-48,5$ & 1,56 & A \\
\hline Distancia sacro-rótula & 50,0 & $47,0-53,1$ & 2,45 & A \\
\hline Perímetro del cráneo & 56,08 & $54,3-57,6$ & 5,41 & RF \\
\hline Perímetro deltoides & 110,2 & 108-112,3 & 3,10 & A \\
\hline Perímetro mesoesternal & 98,1 & $96,2-104,5$ & 1,70 & A \\
\hline $\begin{array}{l}\text { Perímetro abdominal } \\
\text { (cintura) }\end{array}$ & 88,4 & $85,0-95,3$ & 3,84 & $\mathrm{R}$ \\
\hline Perímetro cadera & 91,5 & $89,0-96,1$ & 1,06 & A \\
\hline $\begin{array}{l}\text { Perímetro medio de } \\
\text { brazo relajado }\end{array}$ & 27,6 & 26,9-28,0 & 3,60 & A \\
\hline $\begin{array}{l}\text { Perímetro medio } \\
\text { brazo flexión y tenso }\end{array}$ & 32,4 & $30,1-36,6$ & 2,00 & A \\
\hline Perímetro antebrazo & 26,8 & $26,4-27,0$ & 1,34 & A \\
\hline Perímetro superior muslo & o 55,0 & $54,5-58,4$ & 1,18 & A \\
\hline Perímetro medio pierna & 33,1 & $34,0-35,7$ & 2,12 & A \\
\hline
\end{tabular}

VE: Valores extremos

DEM: Desviación estándar de la media

Prueba Shapiro Wilk:

Nivel de rechazo valor $p<$ dos colas

Representación en la Tabla:

A = Aceptación: $\quad$ Valor x: 0,01 a 0,099

$R=$ Rechazo: $\quad$ Valor $x: 0,10$ a 0,49

$R F=$ Rechazo fuerte: Valor $x:>0,50$ 
Tabla 7. Antropometría del trabajador minero de la altura. Análisis estadístico de la estatura.

\begin{tabular}{cc}
\hline $\mathrm{n}=3000$ & $21-60$ \\
Edad años & \\
Talla centímetros & 151,2 \\
Mínima & 154,6 \\
Cuartil 1 & $\mathbf{1 5 8 , 4}$ \\
Media & 166,7 \\
Cuartil 3 & 169,5 \\
Máxima & 2,37 \\
Desviación estándar & 0,17 \\
Error estándar & \\
Percentiles & 151,1 \\
1 & 153,3 \\
5 & 153,9 \\
10 & 155,2 \\
20 & 156,4 \\
30 & 157,2 \\
40 & $\mathbf{1 5 8 , 4}$ \\
50 & 160,7 \\
60 & 162,4 \\
70 & 164,1 \\
80 & 165,5 \\
90 & 167,8 \\
95 & 169,2 \\
99 &
\end{tabular}

Percentil calculado según: $n=Z^{2} \sigma / 2 d^{2} / e^{2}$

las grandes alturas. Por tanto, quien trabaja en minería es el hombre andino, es decir, el ser humano adaptado a vivir en condiciones fisiológicas diferentes del humano del llano $\left({ }^{20,21}\right)$.

La minería tradicional peruana, que en su mayor parte data de minas y laboreos coloniales, se ubica, sin ser excluyente, en los departamentos de Junín, Cerro de Pasco, Huancavelica, sierra de Lima, Ancash y, en menor grado, La Libertad, Arequipa y Cuzco, zonas que a su vez en la altiplanicie delimitan al grupo étnico quechua, diferenciándolo del grupo aymará, que vive más allá de $\operatorname{los} 3^{\circ} 30^{\prime}$ de latitud sur, y del grupo quechua-mestizo, cuyo hábitat está más acá de los $9^{\circ} 31^{\prime}$ de latitud sur, en los departamentos neo-mineros de Cajamarca y Piura, que tiene mayor proporción de mestizaje con blanco español $\left(^{7,15}\right)$.
En el Perú, son escasos los estudios antropométricos en trabajadores y menos aún en trabajadores andinos, que constituyen la población mayoritaria en la minería del Perú. Por este motivo, quienes trabajamos en salud ocupacional minera sabemos que, en nuestra realidad el trabajador manual, y ahora el intelectual, debe recurrir a modificaciones o adaptaciones de sus herramientas y equipos. Es usual aún ver a obreros 'fabricar' herramientas ad hoc, que las llaman 'hechizas', para poder hacer su trabajo o conducir de pie una pesada retroexcavadora, por no alcanzar los pedales de mando.

A pesar que la minería es la primera contribuidora al PBI peruano $\left({ }^{7}\right)$, para cuidar la salud de los trabajadores mineros no tenemos tablas antropométricas establecidas, que nos ayuden a afrontar los desafíos ergonómicos que la nueva etapa de la modernidad en el trabajo ha comenzado a plantearnos desde el último cuarto del siglo pasado, principalmente en lo referido a evaluación o diseño de puestos de trabajo (22$\left.{ }^{24}\right)$. Y más hoy en día, en que ya no cabe ninguna duda que estas tablas son el método más preciso para conocer las dimensiones corporales de un grupo humano $\left({ }^{25,26}\right)$.

Así pues, en el Perú tenemos necesidad apremiante de investigar, actualizar o complementar las medidas antropométricas de nuestra población laboral en general, pues hasta hoy para tomar alguna decisión ergonómica o inclusive fisiológica, nos basamos en tablas antropométricas que 'estiman' las dimensiones del cuerpo de nuestros trabajadores, 'derivándolas' de tablas de poblaciones extranjeras $\left({ }^{27,28}\right)$.

Nuestra investigación privilegia una visión holística del problema, frente a datos sueltos del hombre de altura estudiados fragmentariamente por diversos autores, principalmente fisiólogos, que por necesidad de su investigación deben valorar algunos parámetros aislados $\left({ }^{29,30-34}\right)$. 

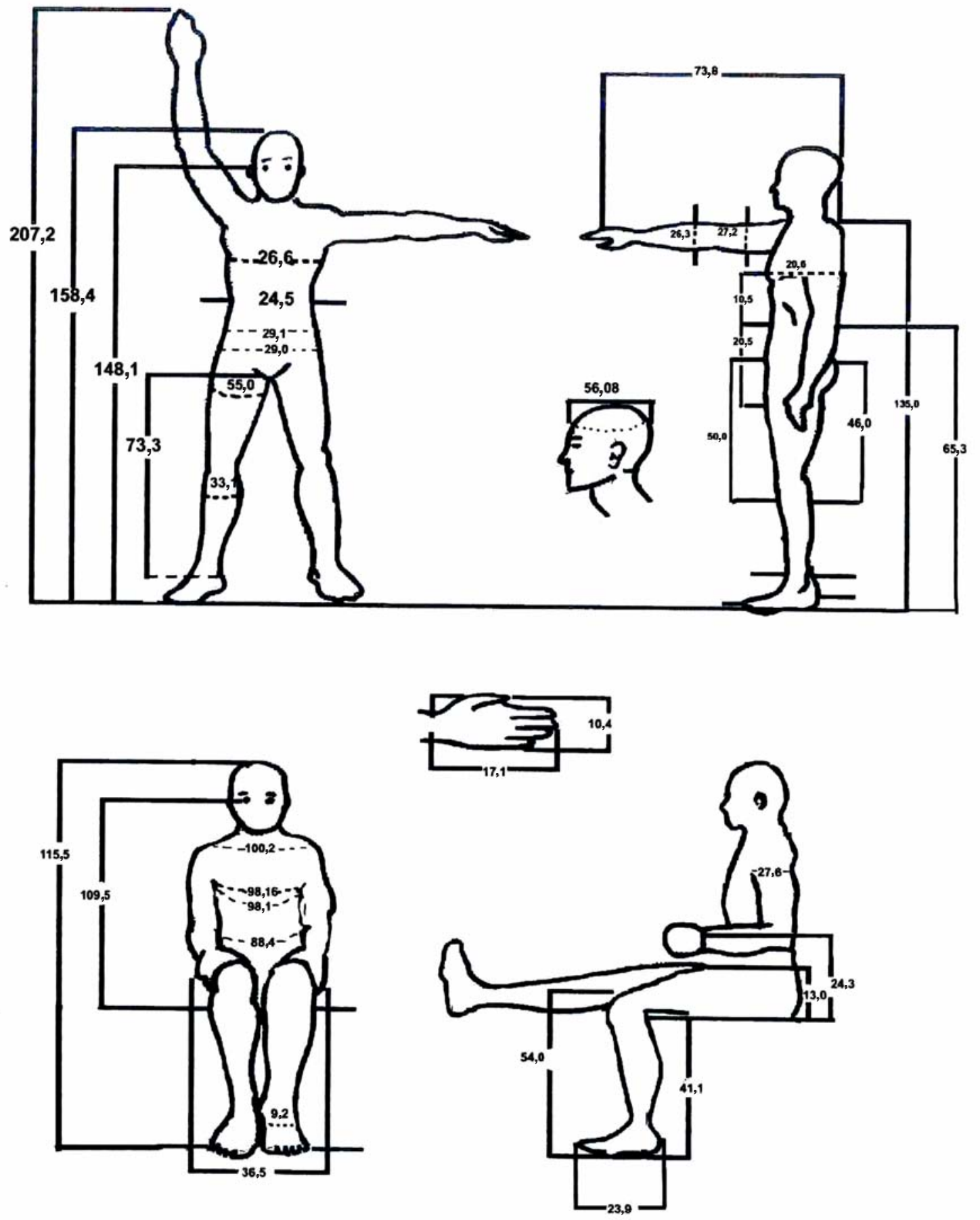

Líneas curvas punteadas: perímetros

Líneas puntadas: diámetros internos

Figura 1.- Evaluación del parámetro talla. 
Los resultados de nuestro estudio confirman los datos de Frisancho, Campos e Iglesias $\left({ }^{29,31}\right)$ y puntualizan, entre otras magnitudes, que el trabajador minero de los Andes centrales del Perú mide en promedio $158,4 \mathrm{~cm}$, con valores extremos (VE) de 151,2 y 169,5 , tiene un peso medio de 58,7 $\mathrm{kg}(\mathrm{VE}=50,4$ y 71,5), que su perímetro torácico en inspiración forzada va de 99,7 a 108,6 , con una media de $105,3 \mathrm{~cm}$, y que su perímetro craneal tiene un promedio de $56,08 \mathrm{~cm}$, valores concordantes con otros estudios peruanos dispersos y con lo encontrado por Santolaya y Blume en trabajadores andinos de Chile $\left({ }^{10,35}\right)$.

Y, aunque no es objetivo del estudio, si comparamos nuestros resultados con los de poblaciones masculinas de trabajadores del llano de otros países, encontramos diferencia en las dimensiones antropométricas, pues éstas son mayores que los de nuestra población andina. Es más, sucede igual con poblaciones laborales que pudiésemos tener como similares -colombianas o mejicanas, por ejemplo $\left({ }^{36,37}\right)$-, por lo que nuestros datos concuerdan con anatomistas y fisiólogos peruanos estudiosos del hombre andino $\left({ }^{31,38}\right)$.

En conclusión, tenemos que la caracterización antropométrica fundamental del trabajador minero andino resalta sus dimensiones propias, que lo hacen ser un modelo humano diferente. Estos resultados obligan a realizar investigaciones integrales en el campo de la antropometría y ergonomía del trabajador peruano en general, a fin de completar su base de referencia antropométrica, por lo que el estudio debería ser ampliado a todos los grupos de trabajadores nativos y residentes de las grandes alturas.

\section{AGRADECIMIENTO}

El autor agradece a su ex empresa Centromín Perú SA el apoyo incondicional en la realización de esta larga investigación. Igualmente, y no menos importante, a sus re- cordados maestros doctores Federico Má A. y César Mayor B. $(\dagger)$, y a todos y cada uno de sus compañeros de trabajo en Centromín y en el Departamento de Medicina Ocupacional CMP La Oroya.

\section{REFERENCIAS BIBLIOGRÁFICAS}

1. Jermain J. Introduction to Physical Anthropology. $7^{\text {th }}$ Ed. Belmont, USA: Wadsworth Publishing Company; 1997.

2. OMS, Comité de Expertos. El Estado físico: uso e interpretación de la antropometría. Ginebra: OMS; 1995. OMS. Informe Técnico 854.

3. McCormick EJ, Sanders MS. Human factors in engineering and design. New York: McGraw Hill; 1987.

4. Clark TS, Corlett EN. The ergonomics of workspaces and machines: a design manual. London: Taylor and Francis; 1984.

5. Bojanini SL. Diseño antropométrico de un puesto de trabajo. Revista Universidad Escuela Administración, Finanzas y Tecnología. Colombia. 1985.

6. Relethford J. The Human Species. $3^{\text {rd }}$ Ed. Mountain View: Mayfield Publishing Company; 1997.

7. Instituto Nacional de Estadística e Informática. Almanaque Estadístico 2000. Lima: INEI; 2001.

8. Monge CC, León Velarde SF, Eds. El Reto fisiológico de vivir en los Andes. Lima: IFEA; 2003.

9. Spielvogel H. Anthropometric of the Andean populations. Instituto Boliviano de Biología de Altura. Bodas de plata 1963-1988. La Paz: IBBA; 1988.

10. Blume FD, Santolaya R, Sherpa M. Anthropometric and lung volume measurements in Himalayan \& Andean natives. J Fases. 1988;2(5):1281.

11. Monge MC. Man, climate, and changes of altitudes. Meteor Monograph. 1954;2(8):50-60.

12. Hurtado A, Velasquez T, Reynafarje C, Lozano R, Aste H, Reynafarje B, et al. Mechanism of natural acclimatization. Studies on the native resident of Morococha Peru at an altitude of 14000 feet. Report to the School of Aviation Medicine. USAF. Randolph Field, 1956.

13. Viault F. Comptes Rendus de Sciences. Paris; 1890. p. 917.

14. Dixon WJ, Massey J. Introduction to Statistical Analysis. New York: McGraw Hill Inc.; 1989.

15. Monge L. Migración e integración. Distribución vertical de la vida, migración y nomadismo. Arch Biol And UNMSM. 1984-1985;13(1-4):13-8.

16. Reynafarge C. La adaptación a las grandes Alturas. Lima: Concytec; 1990.

17. Lohman TG, Roche AF, Martorell R. Anthropometric standardization reference manual. Champaign: Human Kinetics; 1980.

18. Mazza JC. Mediciones antropométricas. Estandarización de las técnicas de medición, actualizada según parámetros internacionales. Revista de Actualización en Ciencias del Deporte. 1993;1(2). 
19. Shapiro SS, Wilk MB. An analysis of variance test for normality. Biometrika. 1965;52:591-611.

20. Frisancho PD, Frisancho VO. Fisiología y Patología en la Altura. Rev Gastroenterol Perú. 1992;12(3):155

21. Baker P, Little M. Man in the Andes. A multidisciplinary study of high altitude Quechua. Pennsylvania, USA: Dowden Hutchinson and Ross Inc.; 1976.

22. Ramírez C. Ergonomía y Productividad. México: Limusa; 2003.

23. Paner J, Zelnik M. Human Dimension and Interior Space. New York, USA: Watson-Guptill Publications; 1979.

24. Mondelo P, Gregori E, de Pedro O, Gómez M. Ergonomía 4: El trabajo en oficinas. Barcelona: Universidad Politécnica de Cataluña; 2001.

25. Pheasant S. Body space. London: Taylor \& Francis; 1995.

26. Paner J, Zelnik M. Las dimensiones humanas en los espacios interiores: estándares antropométricos. Barcelona: Gustavo Gili; 1983.

27. Motmans R, Ceriez E. Ergonomics Research Group. Katholieke Hogeschool. Limburg: Genk; 2005.

28. Ramírez AV, Bejar K, Recabaren A. Alteraciones espirométricas en adolescentes de una ciudad industrial. An Fac Med UNMSM. 2001;62(3):49-58.

29. Frisancho AL, Borkan GA, Klayman JE. Pattern of growth of lowland and highland Peruvian Quechua of similar genetic composition. Human Biologic. 1975;47(3):233.

30. Marticorena E, Severino J, Chávez A. Presión arterial sistémica en el nativo de altura. Arch Inst Biol And. 1967;2:18.

31. Campos J, Iglesias B. Observaciones anatomopatológicas en 49 personas normales, nativos y residentes de altura, muertas en accidentes. Rev Lat Amer Anat Patol. 1957;1:109.
32. Ramirez AV. Efectos de la silicosis sobre la función ventilatoria pulmonar del trabajador minero de las grandes alturas. Rev Bras Saúde Ocupacional 1990;70(18):62-9.

33. Picón Reategui E. Área de superficie corporal y otras medidas antropométricas en nativos de las grandes alturas. Arch de Biolog Andina. 1978;8(1-4):5-17.

34. Hurtado A. Natural acclimatization lo the high altitudes: resident man. En: Cunningham DJC, Lloyd BB, Ed. The regulation of human respiration. London: Blackweil Scientific Publications; 1963. p. 71-82.

35. Santolaya R, et al., Desarrollo minero en la altura, un reto a la medicina actual. Minerales. 1993;48(203):51-62.

36. Estrada MJ. Anthropometric parameters of the working population in Colombia. Rev Fac Nac Salud Pública. 1998;15(2):112-39.

37. Niebel B, Freivalds A, Métodos, estándares y diseño del Trabajo. México: Alfaomega; 2002.

38. Hurtado A. Aspectos fisiológicos y patológicos de la vida en la altura. Lima: Ed. Rimac; 1937.

Manuscrito recibido el 14 de setiembre de 2006 y aceptado para publicación el 30 de octubre de 2006.

Correspondencia:

Augusto V. Ramírez, MD, ACOEM.

Dirección de Salud Ocupacional

Minera Yanacocha

Los Naranjos 139-A.

Cajamarca. Perú.

Correo-e: augustovram@yahoo.es 